INTERNATIONAL DESIGN CONFERENCE - DESIGN 2018

https://doi.org/10.21278/idc.2018.0383

\title{
SUPPORT OF SEARCHING FOR SOLUTIONS BY AUTOMATED STRUCTURAL OPTIMIZATION
}

\author{
A. W. Meyer and S. Vajna
}

\begin{abstract}
In the early stages of product development, engineers search for several different solutions to a design problem, which can then be further detailed and evaluated. This is due to little or fuzzy information about the product at this early phase of product development. The aim of this paper is to support this searching process with automated structural optimization. This is achieved by a combined approach of continuous topology optimization and stochastic algorithms. Computational results are presented and compared with results obtained by pure topology optimization.
\end{abstract}

Keywords: optimisation, early design phase, computational design methods, stochastic algorithms

\section{Introduction and motivation}

Due to globalization, today's companies are under pressure to reduce costs and time for product development and the resulting products. These costs mainly incur in the late stages of the product development process, but they are defined in the early stages. According to Anderson (2014), 80\% of the product costs are determined by the end of the design phase and even $60 \%$ of a product's cumulative lifetime cost are defined by the concept phase. The problem of these early stages is described by the design paradox (Ullman, 2010), shown in Figure 1.

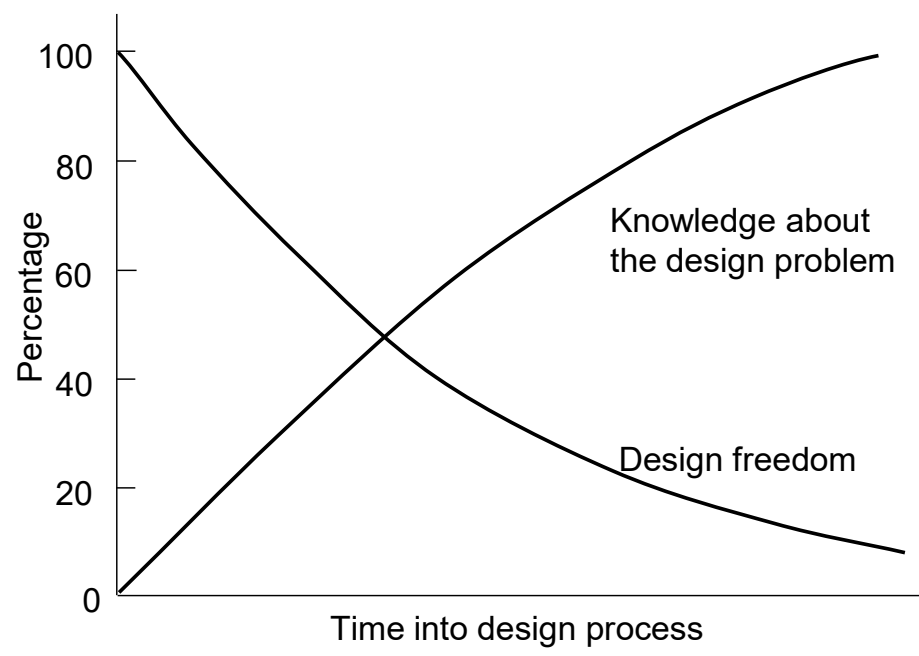

Figure 1. Design paradox (Ullman, 2010) 
In the early stages the engineer has relatively little knowledge about the product, its properties, and the technology later involved. But at the same time a lot of possible design decisions can be taken, which, however, determine the main costs of the product (Chang, 2015). The goal is to collect as much information about the product as early as possible to be able to make changes at the design in the early stages of the product development process when it is less expensive.

One way to overcome the issue of the design paradox is front loading. It is the effort to move the problem identification and solution to the "front" of the process (Thomke and Fujimoto, 2000). Especially the variety of CAx software (e.g. CAD, CAE, CAM software) helps to support this approach (Chang, 2015). By means of product performance simulations, reliability evaluations, manufacturing simulations, and many other tools it is possible to gain more information about the product in the early stages of the development process.

Part of these measures is the automated structural optimization, which supports the engineer in finding suitable component structures, to safely transmit or to support the applied loadings (Bendsøe and Kikuchi, 1988; Bendsøe, 1989; Eschenauer and Olhoff, 2001; Querin et al., 2017). There are three different methods, which are categorized as structural optimization, namely size, shape, and topology optimization. While most applications focus on the ability of these methods to determine the optimal structures, the authors would like to investigate to what extent such algorithms are also suitable for supporting the search for multiple solutions in the early stages of the product development process.

The search for solutions in early stages of product development is described in various process models of design engineering (Hubka, 1973; Ehrlenspiel and Meerkamm, 2013; Feldhusen and Grote, 2013; Jackstien and Vajna, 2014).

In this context, it is worth mentioning the Autogenetic Design Theory (ADT) postulated by Bercsey and Vajna (1994). Within the ADT, procedures, methods, and processes used for the development and adaptation of products can be seen as analogies to the procedures, methods, and processes of biological evolution to create or to adapt individual living beings. As biological evolution does not operate directly on individuals rather on generations of populations the result of this process is always a set of unique individuals, which are of the same value but have different forms and characteristics. Analogous to this, the result of the ADT is ideally a set of equivalent, but not identical solutions that all fulfill the actual state of requirements and conditions best.

Reasons why there is a necessity to create multiple designs with structural optimization in the early stages of design engineering, from which an engineer can choose from, and not just generate one optimized solution, are:

- An automated structural optimization process cannot consider subjective requirements, such as the aesthetic appearance of a product.

- An automated structural optimization cannot always take all requirements to a product into consideration, e.g. manufacturing or thermal requirements.

- In most of the cases, product development is highly iterative due to constantly changing requirements. A set of solutions enables the engineer to react better to changes.

- A set of solutions increases both information and knowledge about an emerging system in the early stages of product development.

\section{Finding the basic approach}

In order to be able to apply structural optimization methods for the search of different structural solutions in the early stages of product development, the approach used must fulfill various requirements. First, it is necessary for the algorithm to be able to produce several different variants. Secondly, it is important that the method is capable to handle models with low detail and little or at times fuzzy information.

The first requirement implies that deterministic algorithms are more or less unsuitable for the search for multiple solutions in product development. The reason for this lies in the intrinsic property of deterministic algorithms to provide identical solutions for the same input variables. In addition, the solution space covered by the individual iteration steps is rather small.

The use of stochastic optimization appears to be much more promising. By using random processes to search the solution space (Arora, 2007), stochastic methods have a higher chance of finding the global optimum. In deterministic methods, this probability is strongly dependent on the starting point of the 
optimization. What is more important for this work, however, is their ability to search a wide area of the solution space and generate a large number of different solutions. This approach, however, results in a higher computational effort. Especially for multi-objective optimization tasks, stochastic methods are able to offer a set of infinite solutions, called the Pareto optimal set (Madeira et al., 2006). The solutions in this set are characterized by the fact that the value of at least one objective can only be reduced if the value of the other objective increases.

Still, stochastic algorithms used in structural optimization usually work with parametric models. These models usually have a high degree of specification and require a high level of knowledge and information.

In order to determine how the second requirement can be fulfilled, different types of structural optimization are examined.

Figure 2 shows three categories of structural optimization, namely size, shape and topology optimization:

- Size optimization: Only the cross sections of the structure are changed (Céa et al., 2000).

- Shape optimization: The optimal form of the oriented boundary curves of the structure's body is searched (Bendsøe, 1995). The insertion of new structural elements such as cavities and struts is excluded (Schumacher, 2013).

- Topology optimization: The optimal distribution of matter in a givens design space is searched.

a)
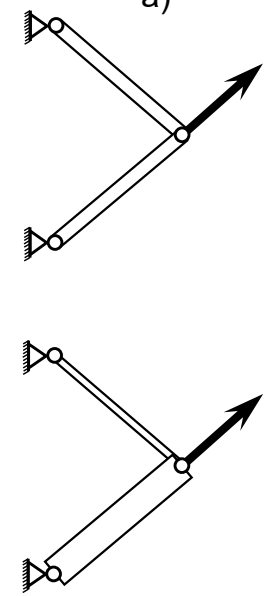

b)
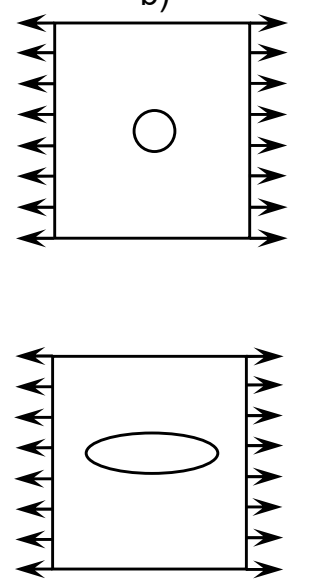

c)
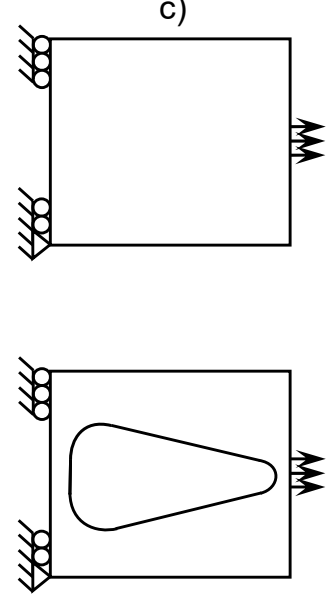

Figure 2. a) Size optimization, b) shape optimization, c) topology optimization (Duysinx, 1996)

Within these three categories of structural optimization, the topology optimization meets the requirements of the early stages of product development best because it requires the least amount of information to be performed. In addition to the information of the design space, the applied loads, and the given boundary conditions, topology optimization doesn't operate on predefined configurations, like size or shape optimization. However, topology optimization usually applies deterministic algorithms, which will not contribute to a large variety of variants.

In order to make use of automated structural optimization for the search of variant solutions in the early stages of the product development process and to avoid the disadvantages of deterministic algorithms a topology optimization method using stochastic algorithms is chosen.

\section{Topology optimization with stochastic algorithms}

The topology of a structure is the arrangement or positioning of its structural elements (Eschenauer and Olhoff, 2001). Accordingly, topology optimization of continuum structures operates by determining for every structural element of the design space whether there is material in the regarded element or not. In the case of FEM discretization, the finite elements represent potential gaps or parts of the supporting structure (Bendsøe, 1995). The aim of this approach is to find the optimum distribution of material 
within a specific domain, taking into account the existing loads and boundary conditions (Garcia-Lopez et al., 2011).

One way to classify the topology optimization is to differentiate between microstructure and macrostructure approaches (Eschenauer and Olhoff, 2001).

The microstructure approaches are based on the principle that the space to be optimized is divided into small areas. Various families of structural topology optimization methods using the microstructure approach have been developed (Bulman et al., 2001). One of the most established families of methods is based on the homogenization method proposed by Bendsøe and Kikuchi (1988), in which the design space is represented by a sponge-like material with infinite micro-scale cells with rectangle holes (Wang and Tai, 2005). Consequently, the optimization algorithm operates on the size and orientation of these holes, which determine the material properties. A disadvantage of this method is the large number of parameters that have to be considered for optimization.

To overcome this, Bendsøe (1989) introduced an approach, which adopts the relative density of each element as the design variable. This approach is called SIMP (Solid Isotropic Micro-structure with Penalization) method (Rozvany et al., 1992) and assumes uniform material properties within each element (Wang and Tai, 2005). The values of the relative density range from 0 (void elements) to 1 (solid element). This raises the issue that values between 0 and 1 are not well transferable to real isotropic material (Rozvany, 2009). In order to circumvent this problem, penalty functions are used that penalize values of relative density between 0 and 1 and thus force the solution to a more feasible design.

In the class of macrostructure approaches, the design space to be optimized is not considered microstructured but as solid isotropic material. Topology optimization is performed in combination with shape optimization and thus the topology of the solid body can be altered by adding or removing material or by inserting holes (Eschenauer and Olhoff, 2001). The method proposed by Eschenauer et al. (1994) applies an alternating procedure of shape optimization and positioning of new holes. These holes are called "bubbles". The shape optimization of these bubbles and of other boundaries of the structure is carried out as a parameter optimization (Schumacher, 2013). To evaluate the solutions of the different iteration steps an automated re-meshing of the structure is required.

In order to overcome local optima and to have a broader scan of the possible solution space, topology optimizations with stochastic algorithms were developed. A large number of these studies use genetic algorithms based on Darwin's theory of evolution by natural selection. These optimization algorithms operate on artificial organisms of a population by means of evolutionary mechanisms (Chapman, 1994). These mechanisms include genetic operators that modify the gene pool of populations that consists of genetic representations of the individual organisms. Every organism represents a solution that is more or less fit to meet the particular problem (Goldberg, 1989; Gen and Cheng, 2000).

The genetic operators are:

- Selection, which picks solutions of the population for reproduction. The fitter the solution, the more likely it is to be picked.

- Crossover, which randomly selects two solutions for recombination to a new organism.

- Mutation, which randomly alters the genome of organisms (Mitchell, 1998).

To examine whether these algorithms can be used for continuous topology optimization, some research has already been carried out (Jensen, 1992; Chapman, 1994; Wang and Tai, 2005; Chen and Chiou, 2013). Most of these methods apply the microstructure approach, where the design space is discretized into finite elements. The design variables are the states of each element, which can contain either material or can be void. This avoids the problem of intermediate densities. With this binary representation each chromosome in a genetic algorithm population controls the material-void states of the elements in the design space (Chapman, 1994). A major task using these approaches is to ensure structural connectivity between the elements with full material. This is related to the problem of checkerboard patterns. Another problem are the computational costs, which depend among other things on the number of design variables. A high number of design variables results in high computational costs and because the number of design variables is equal to the number of elements, genetic algorithms were mostly applied to small models with few elements. 
Similar approaches to perform continuous topology optimization with other stochastic algorithms have been proposed. Algorithms that have also been studied include among others binary particle swarm optimization (Luh et al., 2011), simulated annealing (Bureerat and Limtragool, 2008; Garcia-Lopez et al., 2011), and ant colony optimization (Wu et al., 2009).

In order to take advantage of stochastic algorithms and continuous topology optimization while avoiding the disadvantages, a combined approach was chosen.

\section{The proposed method}

The proposed method uses a combination of macrostructure and microstructure optimization as a new approach not just to find the best solution, but a set of solutions with similar properties but different structural characteristics. This enables the engineer to pick the solutions, which he thinks are worth detailing.

The applied algorithm is composed of an outer and an inner optimization loop. The outer optimization applies a genetic algorithm to execute a parameter optimization of macrostructures to manipulate the design space, whereas the inner optimization uses topology optimization based on the SIMP approach to find optimized structures of this altered design space. Thus, the outer optimization loop forces the topology optimization to find constantly new structures.

In detail, as shown in Figure 3, the algorithms start with the initialization of the first population by randomly generating parameters that determine the shape and position of the macrostructures. In the next step, these macrostructures are injected into the design space and thus create several variants of the design space within the population. Afterwards, continuous topology optimizations are carried out on each individual. Based on these results, the fitness of each individual is assigned. The next step checks if the termination criterion is reached. If this is not the case, a new generation is created based on the previous population by means of the already discussed genetic operators.

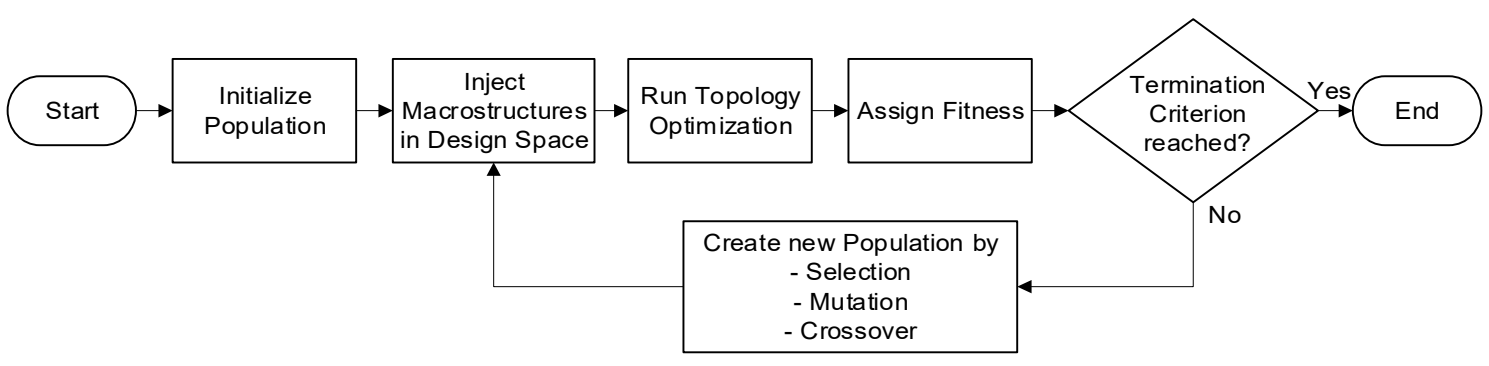

Figure 3. Flow chart of proposed method

\section{Implementation}

To perform the proposed task three already existing software applications operating with numerous file types are used. Table 1 shows a summary of these software applications and what task they perform during the optimization process.

Table 1. Software applications

\begin{tabular}{|l|l|}
\hline Software & Purpose \\
\hline HyperMesh & Meshing, preprocessing \\
\hline OptiStruct & Topology optimization, FE analysis \\
\hline NOA & Parameter optimization using genetic algorithms; management of the evaluation process \\
\hline
\end{tabular}

The starting point of the optimization process is a finite element (FE) model, which contains the discretized unaltered design space, the applied loads, and the boundary conditions. The CAE system Altair HyperMesh is used to build this model and for pre-processing the topology optimization. The FE model is used as a template for all upcoming individuals. 
The software application that is used to perform the parameter optimization using genetic algorithms is called NOA (Natural Optimization Algorithm). NOA supports selective methods of ADT and it can be divided into two parts. The first part is in binary form and generates solutions in the form of parameter combinations. The second part is a batch file that is called from the first part. It executes the various software applications involved and takes care of the different result files that are generated during the process. NOA has been developed at the Chair of Information Technologies in Mechanical Engineering in Magdeburg (Vajna et al., 2007).

Based on the parameters generated by NOA, macrostructures are injected in the design space of the template model of Altair HyperMesh automatically by running scripts using the programming language Tcl (Tool command language) to create the different individuals of a population. The two kinds of macrostructures used in the implemented process are holes and non-design spaces, whereby the nondesign spaces are domains of the model, which are kept unchanged during topology optimization. This leads to the fact that elements of a non-design space contain full material at the end of the optimization. Due to the fact that the injection of the macrostructures operates on the already meshed FE model, a remeshing isn't necessary.

The topology optimization and the following FE analysis are performed by Altair OptiStruct. In the current setting this is done sequentially for each individual of a population, but future parallelization of this process is aimed at (Wünsch, 2017).

\section{Examples and validation}

In order to show that the proposed method is feasible, simple tests were carried out as proof of concept. The model being tested has a two-dimensional square design space, which is fixed at two points and to which a force is applied at the top right corner. The design domain is shown in Figure 4.

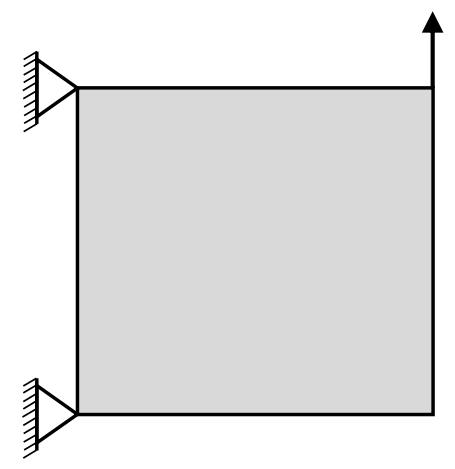

Figure 4. Design domain

At the beginning of the research, a simple topology optimization was conducted, which serves as a reference object for the evaluation of the results of the proposed method. The optimization objective was the minimization of the compliance, with a reduction of the mass by $70 \%$ of the initial mass. Figure 5 shows the resulting structure. In this figure the red areas consist of elements with full material respectively a density of 1 and the blue areas represent void elements with a density of 0 .

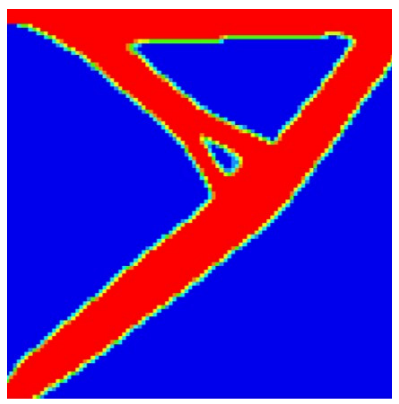

Figure 5. Resulting structure of the topology optimization 
Initially, the proposed method was applied in two test series. At one series, one hole and at the other series one non-design area were injected as macrostructures into the design space. The design variables of the outer optimization (the parameter optimization using a genetic algorithm) are derived by the shape and the position of the respective hole respectively the non-design area. Since both macrostructures are circular, the shape depends only on its radius $r$. The position of the macrostructure is determined by the $\mathrm{x}$ and $\mathrm{y}$ coordinates of its center point. The corresponding coordinate system is located centrally at the design space (Figure 6).

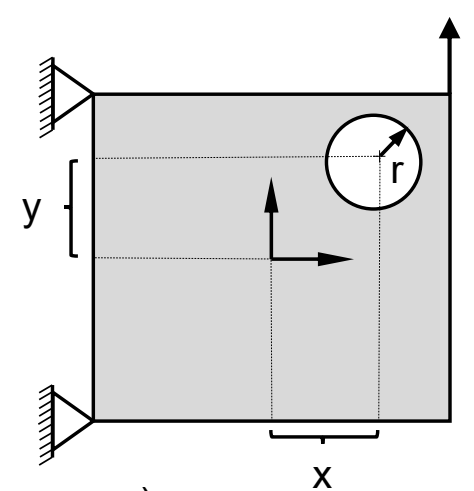

a)

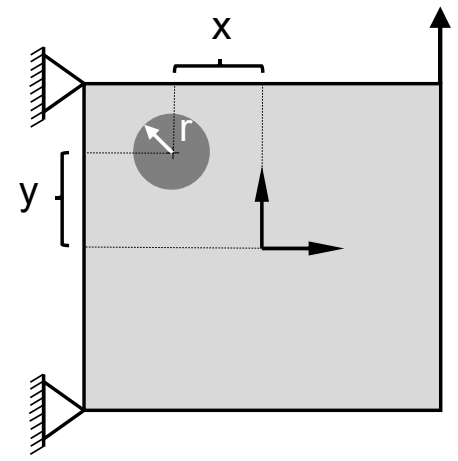

b)

Figure 6. Design space injected by a) a hole and b) a non-design area

In these test series of the proposed method the optimization objective of the outer parameter optimization was also to minimize the compliance of the structure. Both objective and constraints of the inner topology optimization were the same as for the already mentioned pure topology optimization. The resulting solutions of which the structures differ the most from the structure of the pure topology optimization are shown for holes in Figure 7 and for non-design areas in Figure 8.
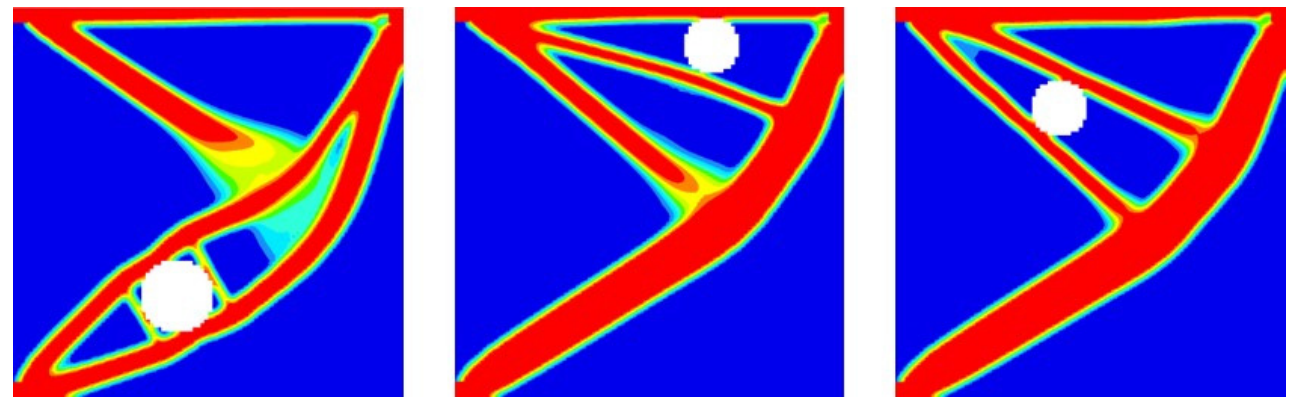

Figure 7. Examples of structural results of optimization using holes as macrostructures
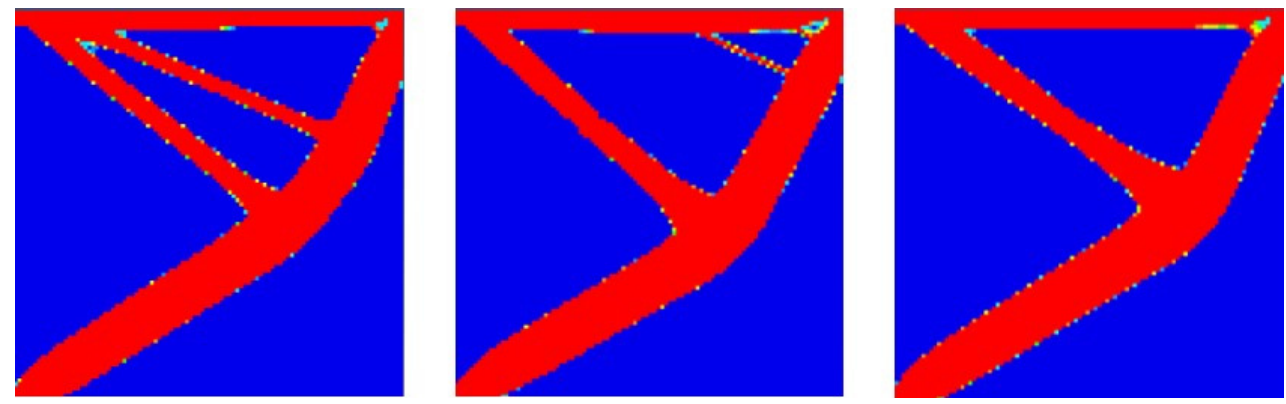

Figure 8. Examples of structural results of optimization using non-design areas as macrostructures 
It can be said that in these experiments the effects of holes in the design space were greater than the effects of non-design areas. One explanation for this is the fact that in many topology optimization solutions the non-design areas were not linked to the load-bearing structure (Figure 9). This was especially the case when the injected macrostructure was too far away from the solution of pure topology optimization.

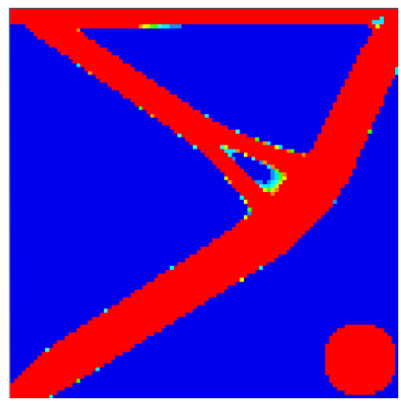

Figure 9. Example of a non-design area not linked to the load-bearing structure

Based on these findings, an optimization was set up that combines both approaches by simultaneously injecting two holes and two non-design areas into the design space. Consequently, the parameter optimization operated with 12 design variables. The population size was set 20 and the maximal number of generations was 200. Figure 10 shows the course of the generation's averaged compliance during the optimization. It shows that the compliance is steadily improving up to the 100th generation. Fluctuations of the graph can be traced back to the random insertion of solutions with very compliant structures into a new generation.

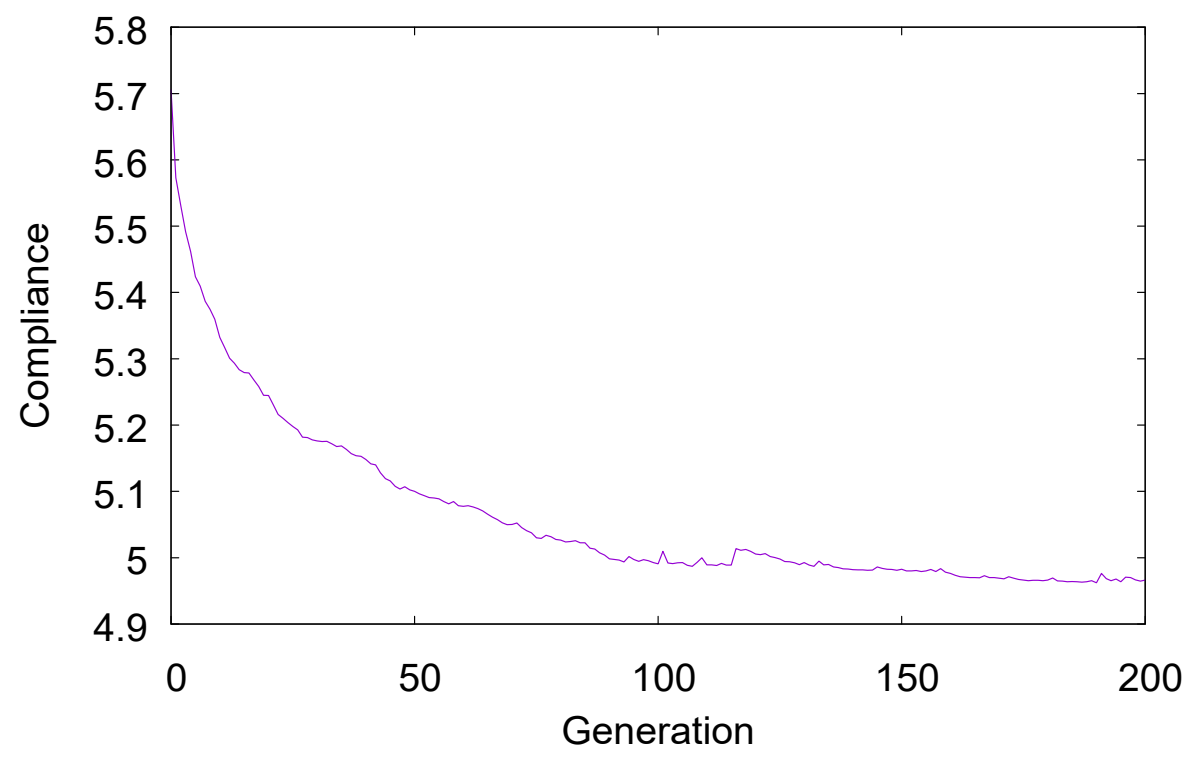

Figure 10. Course of the generation's averaged compliance during optimization

If looking at the results, one can see that the proposed method was able to find solutions that were superior to pure topology optimization. The best solution was $8.44 \%$ less compliant than the reference solution. This implicates that this approach is able to increase the quality of solutions compared to simple topology optimizations. However, it has to be stated that this also linked to much higher computational costs. Figure 11 shows the four best solutions. It is noticeable that these structures are very similar and differ only slightly from the reference solution. 


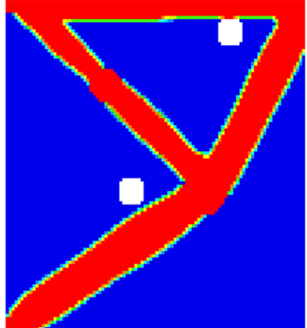

a)

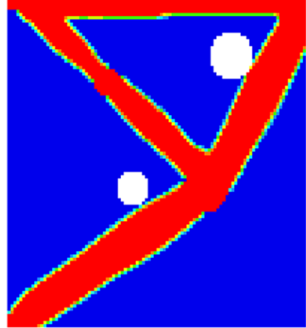

b)

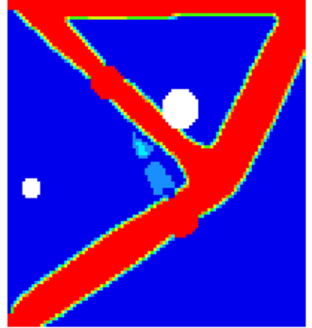

c)

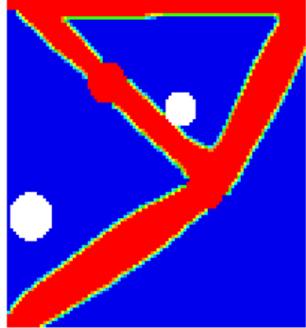

d)

Figure 11. The four best solutions with a) $83.1 \%$, b) $91.6 \%$, c) $91.9 \%$, and d) $91.9 \%$ of the compliance of pure topology optimization

The four most compliant results are shown in Figure 12. All these solutions have in common that a hole was injected near the lower left corner of the design space. Consequently, the resulting structures differ strongly from the best individuals and the reference solution.

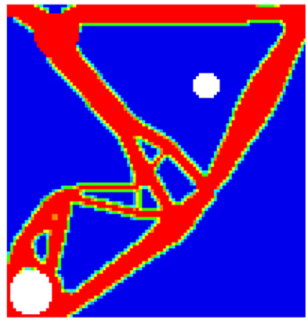

a)

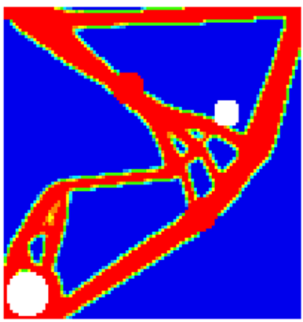

b)

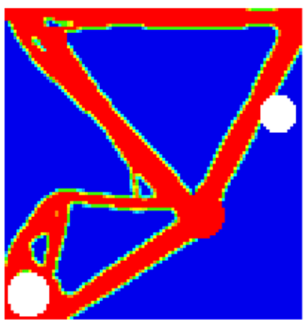

c)

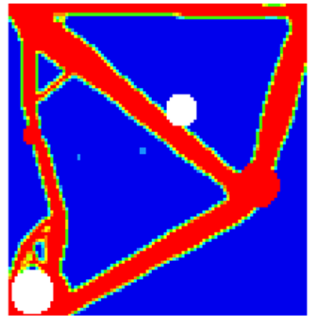

d)

Figure 12. The four worst solutions with a) $154.55 \%$, b) $150.06 \%$, c) $149.91 \%$, and d) $148.55 \%$ of the compliance of pure topology optimization

In the process of the optimization, further unique solutions originated. Figure 13 shows four unique solutions that differ from the reference solution. Furthermore, three of the four structures have a similar compliance than the reference solution. This shows that the approach is capable of generating solutions of the same value but with different form and of providing a set of feasible solutions.

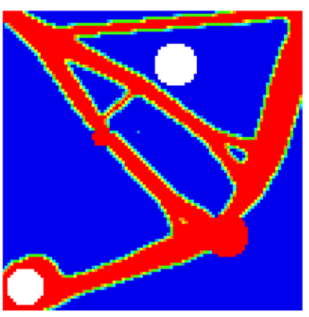

a)

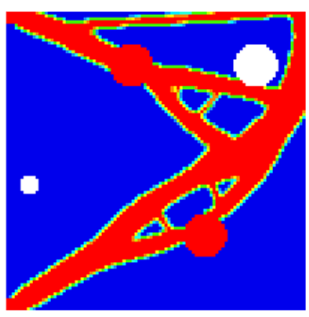

b)

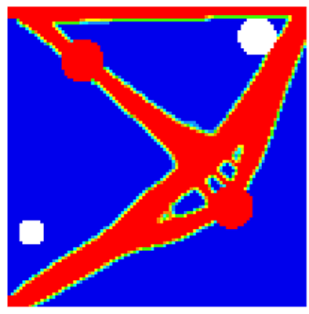

c)

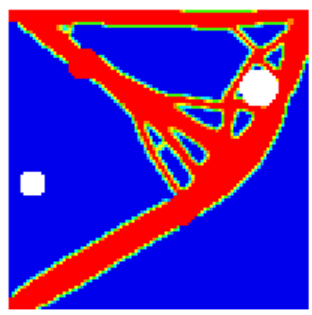

d)

Figure 13. Four unique structures with a) $132.31 \%$, b) $106.83 \%$, c) $98.7 \%$, and d) $100.5 \%$ of the compliance of pure topology optimization

\section{Future work}

The first experiments to validate the proposed method used a simple two-dimensional model with a square design space. This resulted in a simple positioning of the macrostructures, which as circles also had a simple form. The aim of the research is to be able to use the discussed approach with any proposed design space. Consequently, the next research steps will deal with enhancement of the design space to the third dimension. 
In addition, it is worth looking at other shapes of macrostructures, which would require other positioning and shape controlling parameters. Using parameter optimization similar to the bubble method in combination with continuous topology optimization could gain interesting results.

In this approach, a genetic algorithm was used to alter the parameters of the macrostructures. The strategy of using other stochastic algorithms like ant colony optimization, particle swarm, or simulated annealing is worth pursuing.

Furthermore, the experiments have revealed a necessity for the realization as a feasible tool in product development. The algorithm or the user somehow has to be able to evaluate the difference between two structures objectively, not only by the value of the compliance or other mechanical parameters but by a value, that represents the difference between at least two forms of structures. Generally speaking, this value would represent the pure structural diversity of the population. This issue merits further study.

\section{Summary}

This paper presented an approach to support the engineer in finding many structural solutions during the early stages of the product development process by using automated structural optimization. The task was to create a set of various solutions that are roughly equal in value but of different form.

After considering various types of structural optimization, a method was proposed. It uses a combination of parameter optimization with stochastic algorithms and continuous topology optimization. The corresponding framework uses two optimization loops to fulfill the task. The outer loop consists of the parameter optimization, which injects macrostructures into the design space of the FE model, the inner loop runs the topology optimization with this altered design space.

To validate this proposed method various software applications were implemented. In addition, a genetic algorithm was set up to perform the parameter optimization of the outer loop. Furthermore, non-design areas and holes were introduced as possible macrostructures in a two-dimensional design area. The optimization objective of these studies was to minimize the compliance of the structures.

The first results of this method show that using holes as macrostructures has a greater impact on the resulting structures than non-design areas. In addition, the proposed method generated new solutions that differ greatly from the reference structure of the pure topology optimization.

In the end, the capability of this method to produce various solutions with similar value but different form could be confirmed. Although, in regard to the optimization objective most of these solutions were not better than the one based on topology optimization but due to the fuzzy nature of early stages of product development, these solutions don't have to be inferior considering the overall goal of the product development process.

\section{References}

Anderson, D.M. (2014), Design for manufacturability: How to use concurrent engineering to rapidly develop lowcost, high-quality products for lean production, CRC Press, Taylor \& Francis Group, Boca Raton, FL.

Arora, J.S. (2007), Optimization of structural and mechanical systems, World Scientific, Hackensack, NJ. https://doi.org/10.1142/9789812779670

Bendsøe, M.P. (1989), “Optimal shape design as a material distribution problem”, Structural Optimization, Vol. 1 No. 4, pp. 193-202. https://doi.org/10.1007/bf01650949

Bendsøe, M.P. (1995), Optimization of structural topology, shape, and material, Springer, Berlin, Heidelberg. https://doi.org/10.1007/978-3-662-03115-5

Bendsøe, M.P. and Kikuchi, N. (1988), "Generating optimal topologies in structural design using a homogenization method", Computer Methods in Applied Mechanics and Engineering, Vol. 71 No. 2, pp. $197-$ 224. https://doi.org/10.1016/0045-7825(88)90086-2

Bercsey, T. and Vajna, S. (1994), Ein Autogenetischer Ansatz für die Konstruktionstheorie: Beitrag zur vollständigen Beschreibung des Konstruktionsprozesses, Teil 1, CAD-CAM Report, Vol. 2, pp. 66-71.

Bulman, S., Sienz, J. and Hinton, E. (2001), "Comparisons between algorithms for structural topology optimization using a series of benchmark studies", Computers \& Structures, Vol. 79 No. 12, pp. 1203-1218. https://doi.org/10.1016/S0045-7949(01)00012-8

Bureerat, S. and Limtragool, J. (2008), "Structural topology optimisation using simulated annealing with multiresolution design variables", Finite Elements in Analysis and Design, Vol. 44 No. 12-13, pp. 738-747. https://doi.org/10.1016/j.finel.2008.04.002 
Céa, J., Garreau, S., Guillaume, P. and Masmoudi, M. (2000), "The shape and topological optimizations connection", Computer Methods in Applied Mechanics and Engineering, Vol. 188 No. 4, pp. 713-726. https://doi.org/10.1016/S0045-7825(99)00357-6

Chang, K.-H. (2015), Design theory and methods using CAD/CAE, Academic Press, Amsterdam. https://doi.org/10.1016/c2012-0-00835-6

Chapman, C. (1994), Structural Topology Optimization Via the Genetic Algorithm, Master's thesis, Massachusetts Institute of Technology.

Chen, T.Y. and Chiou, Y.H. (2013), "Structural Topology Optimization Using Genetic Algorithms", Proceedings of WCE 2013 / Worlds Congress on Engineering, Vol. 3, London, UK, July 3-5, 2013, pp. 1933-1937.

Duysinx, P. (1996), Optimisation topologique de structure en variables discrètes, PhD thesis, Université de Lièges.

Ehrlenspiel, K. and Meerkamm, H. (2013), Integrierte Produktentwicklung: Denkabläufe, Methodeneinsatz, Zusammenarbeit, Carl Hanser Verlag GmbH \& Co. KG, Munich. https://oi.org/10.3139/9783446436275.fm

Eschenauer, H.A. and Olhoff, N. (2001), "Topology optimization of continuum structures: A review”, Applied Mechanics Reviews, Vol. 54 No. 4, pp. 331-390. https://doi.org/10.1115/1.1388075

Eschenauer, H.A., Kobelev, V.V. and Schumacher, A. (1994), "Bubble method for topology and shape optimization of structures", Structural Optimization, Vol. 8 No. 1, pp. 42-51. https://doi.org/10.1007/bf01742933

Feldhusen, J. and Grote, K.-H. (2013), Pahl/Beitz Konstruktionslehre - Methoden und Anwendung erfolgreicher Produktentwicklung, Springer, Berlin Heidelberg. https://doi.org/10.1007/978-3-642-29569-0

Garcia-Lopez, N.P., Sanchez-Silva, M., Medaglia, A.L. and Chateauneuf, A. (2011), "A hybrid topology optimization methodology combining simulated annealing and SIMP", Computers \& Structures, Vol. 89 No. 15-16, pp. 1512-1522. https://doi.org/10.1016/j.compstruc.2011.04.008

Gen, M. and Cheng, R. (2000), Genetic Algorithms and Engineering Design, John Wiley \& Sons Inc., New York, Chichester.

Goldberg, D.E. (1989), Genetic algorithms in search, optimization, and machine learning, Addison-Wesley, Boston, MA, USA.

Hubka, V. (1973), Theorie der Maschinensysteme, Springer, Berlin, Heidelberg. https://doi.org/10.1007/978-3662-10438-5

Jackstien, K. and Vajna, S. (2014), “Grundlagen des Integrated Design Engineering”, In: Vajna, S. (Ed.) Integrated Design Engineering, Springer, Berlin, Heidelberg, pp. 51-94. https://doi.org/10.1007/978-3-642-41104-5_2

Jensen, E.D. (1992), Topological structural design using genetic algorithms, PhD thesis, Purdue University.

Luh, G.-C., Lin, C.-Y. and Lin, Y.-S. (2011), "A binary particle swarm optimization for continuum structural topology optimization", Applied Soft Computing, Vol.11 No.2, pp. 2833-2844. https://doi.org/10.1016/j.asoc.2010.11.013

Madeira, J.A., Rodrigues, H.C. and Pina, H. (2006), "Multiobjective topology optimization of structures using genetic algorithms with chromosome repairing", Structural and Multidisciplinary Optimization, Vol. 32 No. 1, pp. 31-39. https://doi.org/10.1007/s00158-006-0007-0

Mitchell, M. (1998), An introduction to genetic algorithms, MIT Press, Cambridge, MA, USA.

Querin, O.M., Victoria, M., Alonso, C., Ansola R. and Martí, P. (2017), Topology design methods for structural optimization, Academic Press Inc, London, UK.

Rozvany, G.I.N. (2009), “A critical review of established methods of structural topology optimization”, Structural and Multidisciplinary Optimization, Vol. 37 No. 3, pp. 217-237. https://doi.org/10.1007/s00158-007-0217-0

Rozvany, G.I.N., Zhou, M. and Birker, T. (1992), "Generalized shape optimization without homogenization", Structural Optimization, Vol. 4 No 3-4, pp. 250-252. https://doi.org/10.1007/BF01742754

Schumacher, A. (2013), Optimierung mechanischer Strukturen: Grundlagen und industrielle Anwendungen Springer, Berlin, Heidelberg. https://doi.org/10.1007/978-3-642-34700-9

Thomke, S. and Fujimoto, T. (2000), "The Effect of "Front-Loading" Problem-Solving on Product Development Performance", Journal of Product Innovation Management, Vol. 17 No. 2, pp. $128-142$. https://doi.org/10.1111/1540-5885.1720128

Ullman, D.G. (2010), The mechanical design process, McGraw-Hill, Boston, MA, USA.

Vajna, S., Edelmann-Nusser, J., Kittel, K. and Jordan, A. (2007), "Optimisation of a bow riser using the autogenetic design theory", Journal of Engineering Design, Vol. 18 No. 5, pp. 525-540. https://doi.org/10.1080/09544820701403839

Wang, S.Y. and Tai, K. (2005), "Structural topology design optimization using Genetic Algorithms with a bitarray representation", Computer Methods in Applied Mechanics and Engineering, Vol. 194 No. 36-38, pp. 3749-3770. https://doi.org/10.1016/j.cma.2004.09.003

$\mathrm{Wu}$, C.-Y., Zhang, C.-B. and Wang, C.-J. (2009), "Topology optimization of structures using ant colony optimization", Proceedings of GEC'09 / the 1st ACM/SIGEVO Summit on Genetic and Evolutionary 
Computation, Shanghai, China, June 12-14, 2009, ACM Press, New York, USA, pp. 601-608. https://doi.org/10.1145/1543834.1543916

Wünsch, A. (2017), Effizienter Einsatz von Optimierungsmethoden in der Produktentwicklung durch dynamische Parallelisierung, $\mathrm{PhD}$ thesis, Otto von Guericke University Magdeburg.

Andreas Wilhelm Meyer, Dipl.-Ing.

Otto von Guericke University Magdeburg, Chair of Information Technologies in Mechanical Engineering Universitaetsplatz 2, 39106 Magdeburg, Germany

Email: andreas.meyer@ovgu.de 